\title{
HRAS mutations in bladder cancer at an early age and the possible association with the Costello Syndrome
}

\author{
Willemien Beukers ${ }^{1}$, Aleksander Hercegovac ${ }^{1}$ and Ellen C Zwarthoff $f^{\star}, 1$
}

Bladder tumours of patients $<20$ years have a low incidence of genetic aberrations typically found in tumours in older patients. In this study, we investigated oncogene mutations in patients with bladder cancer $(\mathrm{BC})<20$ years and compared them to older age groups. Interestingly, we observed a relatively high number of HRAS mutations in tumour from young patients. These mutations were also highly uncommon in BCs of older patients, ie, p.(Gly12Ser) and p.(Gly12Ala). Germline mutations in the HRAS gene, especially p.(Gly12Ser/Ala), cause Costello Syndrome (CS), a severe congenital disorder. Indeed, one of the patients had been diagnosed with CS. We hypothesized that some of the other patients might be mosaic for the HRAS mutation and therefore could express some of the clinical features of CS, like tumour predisposition. Hence, we isolated DNA from microdissected stroma and analysed it for HRAS mutations. In the CS patient and in patient $\mathrm{X}$, the mutation was also highly expressed in normal stroma. We conclude that patient $X$ is possibly mosaic for the HRAS mutation. These results suggest that mosaicism for oncogenic HRAS mutations may increase the risk for developing $B C$ at a young age.

European Journal of Human Genetics (2014) 22, 837-839; doi:10.1038/ejhg.2013.251; published online 30 October 2013

Keywords: Urinary Bladder Neoplasms; genes; Ras; mosaicism; Costello Syndrome

\section{INTRODUCTION}

Bladder cancer $(\mathrm{BC})$ is a disease of the elderly with a peak incidence in the sixth decade of life and only $1-2.4 \%$ of all cases present under the age of 40 years. $\mathrm{BC}$ in patients younger than 20 years is even more uncommon with reported incidence rates of only $0.1-0.4 \% .{ }^{1}$ Previous studies demonstrated that tumours of patients $<20$ years lack or have a much lower incidence of epigenetic and genetic aberrations typical of BC in elderly patients. ${ }^{2}$

Together with mutations in FGFR3 and PIK3CA, mutations in the RAS genes are the most common mutations found in $\mathrm{BC}$ and up to $13 \%$ of all bladder tumours harbour a mutation in HRAS, KRAS or NRAS. ${ }^{3}$ There is limited data available regarding these oncogenic mutations in $\mathrm{BC}$ of patients $<20$ years. Therefore, we aimed to investigate FGFR3, PIK3CA, and RAS mutations in $\mathrm{BC}$ of patients $<20$ years and compared the results with the mutation status of a control group consisting of patients with $\mathrm{BC}$ $>20$ years.

\section{MATERIALS AND METHODS}

Fourteen patients < 20 years (median 16.5; range 11-19 years) were included. In addition, three different control groups were investigated, ie, patients diagnosed with BC at $20-40$ years $(n=43), 40-60$ years $(n=45)$, and $>60$ years $(n=58)$. Tumour tissue was obtained from a previous study. ${ }^{4}$ Normal surrounding stroma was microdissected ensuring $>90 \%$ normal cells. Samples were used in accordance with the Dutch Code for 'Proper secondary use of human tissues' (http://www.federa.org). Tumour DNA was analysed for mutations in HRAS, KRAS, and NRAS with a multiplex Snapshot assay as described previously. ${ }^{5}$ This assay identifies $96 \%$ of the RAS mutations in BC, according to the Sanger Institute. In addition, the DNA was screened using the Snapshot analysis for mutations in PIK3CA and FGFR3. ${ }^{5}$ Reference sequences are shown in Supplementary File 1. Sequences and conditions of primers and probes are depicted in Supplementary Tables 1 and 2.

\section{RESULTS}

Table 1 shows the mutations found in the different age groups. FGFR3 and $P I K 3 \mathrm{Ca}$ mutations are more abundant in tumours from patients over 40 years of age. Overall, the FGFR3 mutation co-occurred with a mutation in PIK3CA in 24 cases, similar to previous observations. A mutation in the FGFR3 gene together with a mutation in KRAS was found in only one patient. In the youngest age group, no mutations were found in PIK3CA and only one mutation was observed in the FGFR3 gene. However, 4/14 (29\%) of the young patients harboured a mutation in HRAS, a much higher percentage than in the older age groups (Table 1). Interestingly, not only did we observe this high number of HRAS mutations, but also $3 / 4$ of these mutations were highly uncommon in BC, ie, c.34G $>A$ (p.(Gly12Ser)) and c.35C > G (p.(Gly12Ala)). In addition, another p.(Gly12Ala) mutation was found in a bladder tumour of a 29-year-old patient from the control group. This patient was a known Costello Syndrome (CS) patient. The four patients with the uncommon p.(Gly12Ser/Ala) mutation all had a solitary TaG1 bladder tumour. In these four patients, RAS mutation analysis was performed on DNA isolated from microdissected tumour surrounding stroma. The areas of stroma used as normal surrounding tissue are depicted in Figure 1a. For both tumour and normal tissue, the results of the RAS mutation analysis are shown in Figure 1b. The stroma of patient \#1, a 16-year-old boy with a p.(Gly12Ala) HRAS mutation in his bladder tumour, did also display this HRAS mutation. Likewise, although the signal was low, a p.(Gly12Ser) HRAS mutation was found in the normal stroma of patient \#2, an 18-year-old boy with a p.(Gly12Ser) HRAS mutation in his bladder tumour. There was no mutation found in the normal tissue of patient \#3, a 16-year-old male. In the 29-year-old female CS patient (Patient \#4) from the control group, the p.(Gly12Ser) HRAS mutation was also found in the surrounding stroma. 
Table 1 Distribution of oncogene mutations in bladder tumours of different age categories

\begin{tabular}{|c|c|c|c|c|}
\hline & \multicolumn{4}{|c|}{ Age categories } \\
\hline & $<20$ Years & $20-40$ Years & $40-60$ Years & $>60$ Years \\
\hline & $\mathrm{n}=14$ & $\mathrm{n}=43$ & $\mathrm{n}=45$ & $\mathrm{n}=58$ \\
\hline \multicolumn{5}{|l|}{ HRAS } \\
\hline p.(Gly12Ala) & 1 & & & \\
\hline p.(Gly12Ser) & 2 & 1 & & \\
\hline p.(Gln61Leu) & & 2 & & 1 \\
\hline p.(Gln61Arg) & 1 & 1 & 1 & 1 \\
\hline \multicolumn{5}{|l|}{ KRAS } \\
\hline p.(Gly12Cys) & & 1 & 2 & 1 \\
\hline p.(Gly12Asp) & & 1 & 1 & 1 \\
\hline p.(Gly372Cys) & & & 4 & 1 \\
\hline p.(Ser249Cys) & 1 & 12 & 18 & 26 \\
\hline p.(Tyr375Cys) & & & 8 & 5 \\
\hline p.(Arg248Cys) + p.(Ser249Cys) & & & & 1 \\
\hline Wild type & 13 & 29 & 13 & 21 \\
\hline Unknown & & 1 & & \\
\hline \multicolumn{5}{|l|}{ PIK3CA } \\
\hline p.(Glu542Lys) & & 2 & 2 & 3 \\
\hline p.(Glu545Gly) & & & 1 & 1 \\
\hline p.(Glu545Lys) & & 1 & 6 & 8 \\
\hline p.(His1047Arg) & & & 2 & 2 \\
\hline HRAS and PIK3CA mutation & & & & 1 \\
\hline FGFR3, KRAS, and PIK3CA mutation & & & 1 & 1 \\
\hline
\end{tabular}

\section{DISCUSSION}

$\mathrm{BC}$ of patients $<20$ years seem to form a molecular distinct group compared with BC of the elderly. In this study, we screened BC of patients $<20$ years for oncogenic mutations that are typically found in $\mathrm{BC}$ of the elderly. As expected, no mutations were found in the PIK3CA gene, and only one mutation in the FGFR3 gene. However, a high amount of uncommon p.(Gly12Ser/Ala) HRAS mutations were observed in the youngest age category.

Germline mutations in the HRAS gene are found in several developmental disorders, such as the CS. CS is a rare syndrome with only 300-400 reported cases worldwide. CS is caused by point mutations in the HRAS gene, mostly resulting in p.(Gly12Ser) or p.(Gly12Ala). This multisystem disorder causes mental retardation, distinctive facial appearance, cardiovascular abnormalities, skin and musculoskeletal abnormalities, and tumour predisposition. Most frequent malignancy found is the embryonal rhabdomyosarcoma, but also cases with $\mathrm{BC}$ have been described. ${ }^{6}$ It appeared that the 29-year-old patient with a p.(Gly12Ser) HRAS mutation in the bladder tumour was known to have CS and therefore the p.(Gly12Ser) HRAS mutation could also be observed in the normal surrounding stroma. The mutation found in the normal tissue of patient \#2 could be due to contamination of tumour cells, as the signal is mutant peak is low compared with the wild-type peak. However, the mutation signal in the normal tissue of patient \#1 is too high to be caused by contamination of tumour cells during microdissection. This suggests that this patient could be mosaic for the p.(Gly12Ala) HRAS mutation and therefore might have some of the clinical features of CS, such as tumour predisposition. Blood or other patients' tissue samples would be necessary to strengthen this hypothesis. Unfortunately, because of ethical limitations in accessing clinical data, we were unable to obtain additional tissue or information on possible other CS-like features of patients \#1 (and patient \#2).

Mosaicism of HRAS mutations in relation with CS was described previously in a small number of patients. ${ }^{7,8}$ The phenotype of patients with mosaicism may differ and the clinical manifestations could occur along a spectrum depending on the affected tissue compartments. ${ }^{9}$ 
a

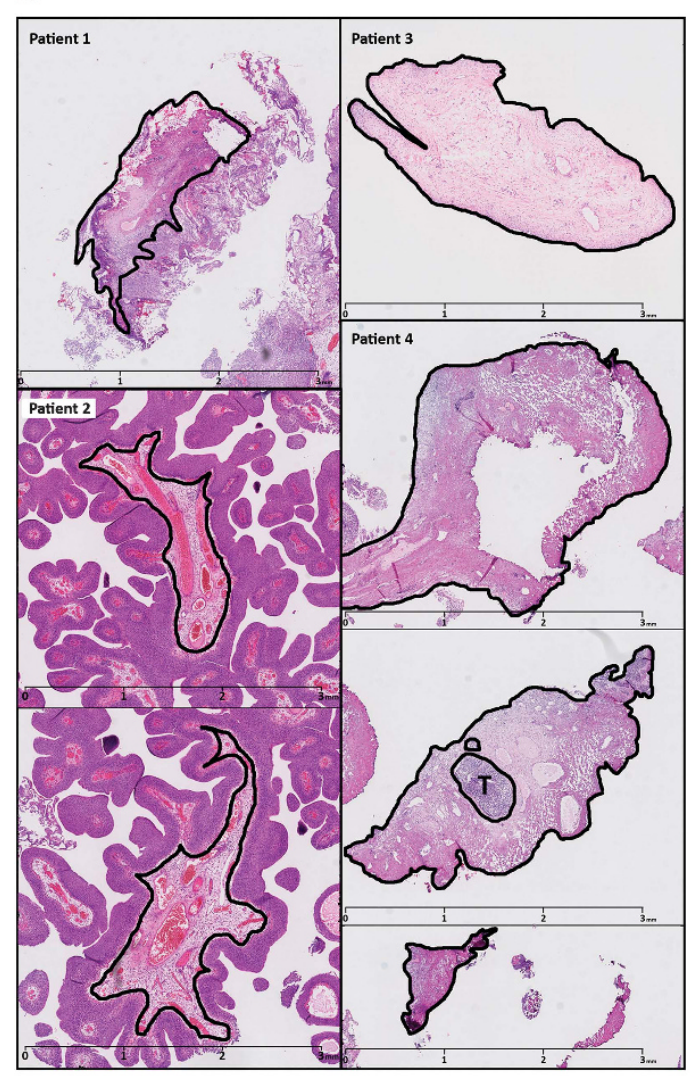

b
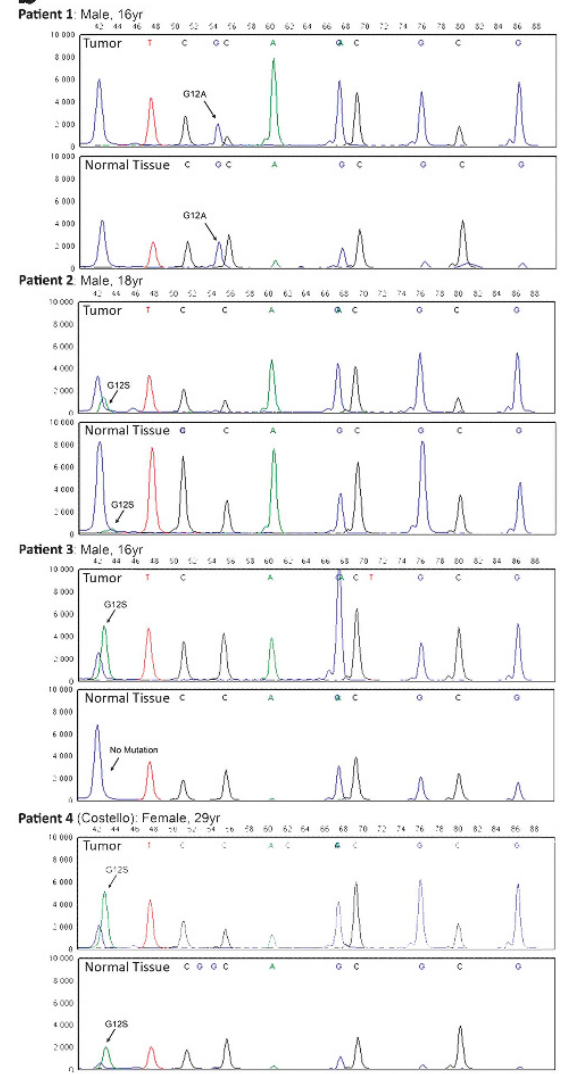

Figure 1 (a) Microdissected areas used as normal surrounding tissue in the Snapshot analysis. Microdissection ensured $>90 \%$ normal stromal cells. Stroma from patient \#1,2, and 4 was derived from the same side as the bladder tumour. Stroma from patient \#3 was obtained from random bladder biopsies. (b) RAS Snapshot analysis of tumour tissue and normal surrounding stroma of four patients. T, tumour tissue.

In a recently published case report, mutant HRAS mosaicism was also described in relation to BC. The publication described a patient with an extensive mosaicism for a HRAS p.(Gly12Ser) mutation. This 49-year-old man presented with widespread congenital epidermal nevi, multiple bladder tumours, and a BC lung metastasis. The HRAS p.(Gly12Ser) mutation was found in the epidermal nevi as well as in the multiple bladder tumours and lung metastasis. ${ }^{10}$ The authors concluded that these findings corroborate the theory that RAS mutations may occur during early embryogenesis and could contribute to carcinogenesis at later age. Our results contribute to this hypothesis and suggest that mosaicism for oncogenic HRAS mutations may even increase the risk for developing BC at very young age. Thus, RAS mutation analysis should be performed on bladder tumours of young patients and if positive, the patient should be screened for possible manifestations of CS.

\section{CONFLICT OF INTEREST}

The authors declare no conflict of interest.

\section{ACKNOWLEDGEMENTS}

We thank the Department of Pathology of the Leiden University Medical Centre and the PALGA-foundation for providing them with tumour material. This study was supported by Erasmus MC (MRace 2007); European Community Seventh Framework program FP7/2007-2012, and grant agreement no 201663.

1 Javadpour N, Mostofi FK: Primary epithelial tumours of the bladder in the first two decades of life. J Urol 1969; 101: 706-710.

2 Wild PJ, Giedl J, Stoehr R et al: Genomic aberrations are rare in urothelial neoplasms of patients 19 years or younger. J Pathol 2007; 211: 18-25.

3 Jebar AH, Hurst CD, Tomlinson DC, Johnston C, Taylor CF, Knowles MA: FGFR3 and Ras gene mutations are mutually exclusive genetic events in urothelial cell carcinoma. Oncogene 2005; 24: 5218-5225.

4 Beukers W, Hercegovac A, Vermeij M et al: Hypermethylation of the Polycomb Group Target Gene PCDH7 in Bladder Tumours from Patients of all Ages. J Urol 2013; 190: 311-316.

5 Kompier LC, Lurkin I, van der Aa MN, van Rhijn BW, van der Kwast TH, Zwarthoff EC: FGFR3, HRAS, KRAS, NRAS and PIK3CA mutations in bladder cancer and their potential as biomarkers for surveillance and therapy. PLoS One 2010; 5: e13821.

6 Hennekam RC: Costello syndrome: an overview. Am J Med Genet C Semin Med Genet 2003; 117C: 42-48.

7 Gripp KW, Stabley DL, Nicholson L, Hoffman JD, Sol-Church K: Somatic mosaicism for an HRAS mutation causes Costello syndrome. Am J Med Genet A 2006; 140: 2163-2169.

8 Sol-Church K, Stabley DL, Nicholson L, Gonzalez IL, Gripp KW: Paternal bias in parental origin of HRAS mutations in Costello syndrome. Hum Mutat 2006; 27: 736-741.

$9 \mathrm{Hamm} \mathrm{H}$ : Cutaneous mosaicism of lethal mutations. Am J Med Genet 1999; 85 : 342-345.

10 Hafner C, Toll A, Real FX: HRAS mutation mosaicism causing urothelial cancer and epidermal nevus. N Engl J Med 2011; 365: 1940-1942.

Supplementary Information accompanies this paper on European Journal of Human Genetics website (http://www.nature.com/ejhg) 